\title{
IMPLICITLY DEFINED MAPPINGS IN LOCALLY CONVEX SPACES
}

\author{
TERRENCE S. MCDERMOTT
}

\begin{abstract}
Results on existence, uniqueness, continuity and differentiability of implicit functions in locally convex, linear topological spaces are obtained, and certain of these results are applied to obtain results on the existence and continuous dependence on parameters of global solutions for a nonlinear Volterra integral equation.
\end{abstract}

Introduction. So far, no single theory of differentiation or local linear approximation of nonlinear mappings between locally convex, linear topological spaces seems to have clearly established a place of preeminence. In particular, none has produced an implicit function theorem of anywhere near the comprehensive character of the results available in the context of Banach spaces (see, for example, [3]). Results in more general settings can be found in [1], [4], [5], and [6].

In this article, we discuss the problem of implicit functions in locally convex spaces in light of the ideas and results developed in [11]. In $\$ 1$ we give conditions for the existence of an implicit function, then obtain results dealing with its uniqueness and continuity. In $\$ 2$ certain results from the first are applied to discuss global, continuous solutions for a nonlinear, Volterra integral equation. Finally, in $\$ 3$ we give conditions under which a differentiable implicit function will exist, differentiability being understood in the sense of Sebastião è Silva [14].

For the convenience of the reader, $\$ 0$ has been included containing the essential definitions and results from [11] that are used in the present work.

0. Preliminaries. In this section, we shall state the basic definitions and results from [11] that will be needed in the present work. Throughout the section, let $E$ and $F$ denote real, locally, convex, linear topological spaces.

If $A$ is an absolutely convex set in $E$, we denote by $E_{A}$ the linear subspace of $E$ generated by $A$. The functional $\|\cdot\|_{A}$ defined on $E_{A}$ by $\|x\|_{A}=\inf \{\lambda>0: x \in \lambda A\}$ is a norm on $E_{A}$ in case $E$ is Hausdorff and $A$ is bounded. Further, $\|x\|_{\alpha A}=(1 / \alpha)\|x\|_{A}$ for any $\alpha>0$. We assume henceforth that all spaces are Hausdorff.

Received by the editors June 1, 1970 and, in revised form, December 15, 1970.

AMS 1970 subject classifications. Primary 47H15; Secondary 46A05, 58C15.

Key words and phrases. Implicit function, locally convex, linear topological space, nonlinear Volterra integral equation, differentiability.

(1) The main results of $\$ 1$ and 2 of this article were essentially contained in the author's doctoral dissertation completed in 1969 at the University of Southern California under the guidance of Dr. Donald H. Hyers.

Copyright (C) 1971, American Mathematical Society 
Definition 0.1. A class $\mathscr{B}$ of subsets of $E$ shall be called a bounded covering for $E$ if the following three conditions hold:

(C1) Each $B \in \mathscr{B}$ is bounded and absolutely convex.

(C2) If $B \in \mathscr{B}$, then $\lambda B \in \mathscr{B}$ for all $\lambda \geqq 1$.

(C3) $\bigcup_{B \in \mathscr{B}} B=E$.

DEFINITION 0.2 . Let $\mathscr{B}$ be a bounded covering for $E$ and let $x_{0}$ be an element of an open set $N \subset E$. Suppose $U$ is an absolutely convex neighborhood of 0 in $E$ and $\delta>0$ is a given real number. Further, assume $f: N \rightarrow F$ is a not necessarily linear map. A linear map $L: E \rightarrow F$ will be said to $(\delta, \mathscr{B})$-approximate $f$ at $x_{0}$ on $U$ if the following four conditions hold:

(A1) $x_{0}+U \subset N$.

(A2) $L(B)$ is bounded in $F$ for every $B \in \mathscr{B}$.

(A3) $(f-L)\left(x_{0}+(B \cap U)\right) \subset(f-L) x_{0}+F_{L(B \cap U)}$ for all $B \in \mathscr{B}$.

(A4) For each $B \in \mathscr{B}$,

$$
\left\|(f-L) x_{1}-(f-L) x_{2}\right\|_{L(B \cap U)} \leqq \delta\left\|x_{1}-x_{2}\right\|_{B \cap U}
$$

for all $x_{1}$ and $x_{2}$ in $x_{0}+(B \cap U)$.

THEOREM 0.1. Suppose $E$ is sequentially complete and $\mathscr{B}$ is a bounded covering for $E$. Let $N$ be an open neighborhood of $x_{0} \in E$ and assume $f: N \rightarrow F$ is continuous in $N$. If for some $\delta, 0<\delta<1$, there is an open linear mapping $L$ of $E$ onto $F$, having closed null space, which $(\delta, \mathscr{B})$-approximates $f$ at $x_{0}$, then there exists an absolutely convex neighborhood $U$ of 0 in $E$ such that $x_{0}+U \subset N$, and $f\left(x_{0}+U\right) \supset f\left(x_{0}\right)+V$, where $V$ is a neighborhood of 0 in $F$. In fact, $V=L((1-k) U / 4), k=2 \delta /(1+\delta)$.

Definition 0.3. A bounded covering $\mathscr{B}$ for $E$ is said to be pair-containing if for each pair of points $x_{1}$ and $x_{2}$ in $E$, there is a $B \in \mathscr{B}$ such that $x_{1} \in B$ and $x_{2} \in B$.

COROLlARY 0.1. In the theorem, if $L$ is assumed to be injective, and $\mathscr{B}$ is assumed to be pair-containing, then for each $y \in f\left(x_{0}\right)+V$, there is a unique $x \in x_{0}+U$ such that $y=f(x)$.

Proposition 0.1. L: $E \rightarrow F$ is injective implies that for every bounded absolutely convex set $A \subset E$,

$$
\left\|L x_{1}-L x_{2}\right\|_{L(A)}=\left\|x_{1}-x_{2}\right\|_{A}
$$

for all $x_{1}$ and $x_{2}$ in $A$.

If $B$ is an absolutely convex, bounded set in $E$, and $U$ an absolutely convex neighborhood of 0 in $E$, it is easy to verify that $E_{B \cap U}=E_{B}$. This fact is used in the computations of $\S 2$.

1. An implicit function theorem. Throughout this section, let $G$ be an arbitrary topological space, $E$ a sequentially complete Hausdorff, locally convex, linear topological space and $F$ a Hausdorff, locally convex, linear topological space. 
THEOREM 1.1. Let $K$ be an open subset of $G$ containing $y_{0}, N$ an open neighborhood of 0 in $E$ and $f: K \times N \rightarrow F$ a map satisfying $f\left(y_{0}, 0\right)=0$. Suppose that for each $y$ in $K$, the map $f_{y}: E \rightarrow F$ defined by $f_{y}(x)=f(y, x)$ is $\left(\delta_{y}, \mathscr{B}_{y}\right)$-approximable at 0 on $U_{y}$ by an open, onto linear map $L_{y}: E \rightarrow F$, having closed null space, where $0<\delta_{y}<1$, and $\mathscr{B}_{y}$ is a bounded covering for $E$. Suppose, in addition, that $g: K \rightarrow F$ defined by $g(y)=f(y, 0)$ is continuous at $y_{0}$. If for each $y$ in $K, f_{y}$ is continuous on $N$, and the set

$$
W=\bigcap_{y \in K} L_{y}\left(\left(1-k_{y}\right) U_{y}\right), \quad k_{y}=2 \delta_{y} /\left(1+\delta_{y}\right),
$$

is a neighborhood in $F$, then there is a neighborhood $V$ of $y_{0}$ in $K$ and a neighborhood $U$ of 0 in $N$ and a map $\varphi: V \rightarrow U$ such that $f(y, \varphi(y))=0$ for all $y$ in $V$, and $\varphi\left(y_{0}\right)=0$.

Proof. From Theorem 0.1 , we know that $f(y, 0)+L_{y}\left(\left(1-k_{y}\right) U_{y} / 4\right)$ is covered by $f_{y}\left(U_{y}\right)$ for each $y \in K$. Let $W^{\prime}=\frac{1}{4} W$. Since $g$ is continuous at $y_{0}$, there is a neighborhood $V$ of $y_{0}$ in $K$ such that $f(V, 0) \subset W^{\prime}$. We conclude that for each $y \in V$, $f(y, 0)+W^{\prime}$ contains 0 , because $(y, 0) \in V \times\{0\}$, and $W^{\prime}$ is balanced, thereby containing $-f(y, 0)$ as well as $f(y, 0)$. Furthermore, $f(y, 0)+W^{\prime} \subset f(y, 0)$ $+L_{y}\left(\left(1-k_{y}\right) U_{y} / 4\right)$ for each $y \in V$. Hence, we can find for each $y \in V$ a point $x=\varphi(y)$ in $U_{y}$ such that $f(y, \varphi(y))=0$. Setting $\varphi\left(y_{0}\right)=0$ and $U=\bigcup_{y \in V} U_{y}$, we have the result.

COROLlaRY 1.1. Carrying over the hypotheses and notation of the theorem, assume in addition that for each $y \in K, L_{y}$ is bijective, and $\mathscr{B}_{y}$ is pair-containing. Then, there is a unique map $\varphi: V \rightarrow U$ having the property that $\varphi(y) \in U_{y}$ and satisfying $f(y, \varphi(y))=0$.

Proof. The hypotheses allow us to employ Corollary 0.1 in place of Theorem 0.1 , concluding thereby that for each $y$ in $V$, there is a unique $x=\varphi(y)$ in $U_{y}$ with $f(y, \varphi(y))=0$. In particular, then, $\varphi\left(y_{0}\right)$ must be 0 .

REMARK 1.1. We note here for reference that the neighborhood $V$ on which $\varphi$ is defined may be taken to be any neighborhood of $y_{0}$ in $K$ satisfying $f(V, 0) \subset W$.

THEOREM 1.2. Let $K \subset G$ and $N \subset E$ be open neighborhoods of $y_{0}$ and 0 respectively, and let $f: K \times N \rightarrow F$ be continuous with $f\left(y_{0}, 0\right)=0$. Suppose there is a linear homeomorphism $L: E \rightarrow F$, a real number $\delta, 0<\delta<1$, and a bounded covering $\mathscr{B}$ for $E$ such that $L(\delta, \mathscr{B})$-approximates $f_{y}$ at $x=0$ on $U$ for all $y$ in $K$, where $U$ is an absolutely convex neighborhood of 0 in $E$. If $\mathscr{B}$ is pair-containing, and the set

$$
S=\left\{L^{-1}(f(y, 0)): y \in K\right\}
$$

is a subset of some set $B \in \mathscr{B}$, then there exists a unique continuous map $\varphi: V \rightarrow U$ such that $f(y, \varphi(y))=0$, where $V$ is chosen according to Remark 1.1 .

Proof. The hypotheses of Theorem 1.1 and its corollary are evidently satisfied. Hence, the existence and uniqueness of $\varphi$ are automatic. To see that $\varphi$ is continuous, 
we must refer to the construction of Theorem 3 of [11]. From it we can deduce easily that for each $y \in V, \varphi(y)$ is the limit of the sequence $x_{n}(y)$ given by

$$
\begin{aligned}
& x_{1}(y)=-L^{-1}(f(y, 0)), \\
& x_{n}(y)=x_{n-1}(y)-L^{-1}\left(f\left(y, x_{n-1}(y)\right)\right), \quad n>1 .
\end{aligned}
$$

The condition that $S$ be contained in some set $B \in \mathscr{B}$ allows us to apply Remark 2 of [11] to conclude $\forall y \in V$, that $x_{n}(y) \in B \cap U$ for all $n \geqq 1$. In the proof of Theorem 3 referred to above, the sequence $x_{n}$ is shown to be Cauchy with respect to $\|\cdot\|_{B \cap U}$. Since $B \cap U$ is a bounded set, the sequence $x_{n}(y)$ is uniformly Cauchy with respect to the topology of $E$, hence uniformly convergent to $\varphi(y)$ on $V$. But the continuity of $f$ and $L^{-1}$ implies that each $x_{n}(y)$ is continuous. Hence, $\varphi$ is the uniform limit of continuous functions and is therefore continuous.

The conditions of the last theorem result in $\varphi(V)$ being a bounded set in $E$ (since $\varphi(V) \subset \bar{B})$. The next result is of a different character.

THEOREM 1.3. Let $K \subset G$ and $N \subset E$ be open neighborhoods of $y_{0}$ and 0 respectively, and let $f: K \times N \rightarrow F$ be continuous with $f\left(y_{0}, 0\right)=0$. Suppose there is a base $\mathscr{N}$ of absolutely convex neighborhoods of 0 in $E$ so that for each $U \in \mathscr{N}$ there exists a $\delta_{U}, 0<\delta_{U}<1$, such that for every $y \in K$ there is a linear homeomorphism $L_{y}: E \rightarrow F$ and a pair-containing, bounded covering $\mathscr{B}_{y}$ for $E$ such that $L_{y}\left(\delta_{U}, \mathscr{B}_{y}\right)$-approximates $f_{y}$ at 0 on $U$. If, for every $U \in \mathscr{N}, W_{U}=\bigcap_{y \in K} L_{y}(U)$ is a neighborhood in $F$, then for each $U \in \mathscr{N}$ there exists a neighborhood $V$ of $y_{0}$ in $K$ and a unique map $\varphi: V \rightarrow U$ satisfying $f(y, \varphi(y))=0$. In addition, $\varphi$ is continuous at $y_{0}$.

Proof. Choose $U \in \mathscr{N}$. The hypotheses of Corollary 1.1 are easily seen to be satisfied with $\delta_{y}=\delta_{U}$ and $U_{y}=U$ for all $y \in K$. Hence, there is a unique map $\varphi: V \rightarrow U, V$ as in the corollary. To see $\varphi$ is continuous at $y_{0}$, let $S$ be any neighborhood of $\varphi\left(y_{0}\right)=0$ in $E$. Find $U^{\prime} \in \mathscr{N}$ such that $U^{\prime} \subset S \cap U$. Applying the corollary again, we find a neighborhood $V^{\prime}$ of $y_{0}$ and a unique map $\varphi^{\prime}: V^{\prime} \rightarrow U^{\prime}$ such that $f\left(y, \varphi^{\prime}(y)\right)=0$. But $\varphi(y)=\varphi^{\prime}(y)$ for all $y \in V \cap V^{\prime}$. In fact, if $y \in V \cap V^{\prime}, \varphi(y)$ and $\varphi^{\prime}(y)$ are in $U$, and there is a $B \in \mathscr{B}_{y}$ containing both $\varphi(y)$ and $\varphi^{\prime}(y)$. But then we have

$$
\left\|f\left(y, \varphi^{\prime}(y)\right)-f(y, \varphi(y))-L_{y}\left(\varphi^{\prime}(y)-\varphi(y)\right)\right\|_{L_{y}(B \cap U)} \leqq \delta_{U}\left\|\varphi^{\prime}(y)-\varphi(y)\right\|_{B \cap U} .
$$

Using the fact that $L_{y}$ is injective through Proposition 0.1 , the fact that $\delta_{U}<1$, and that $f(y, \varphi(y))=f\left(y, \varphi^{\prime}(y)\right)=0$, we see that $\varphi(y)=\varphi^{\prime}(y)$. Hence, we can conclude that $\varphi\left(V \cap V^{\prime}\right) \subset U^{\prime} \subset S$. Hence, $\varphi$ is continuous at $y_{0}$.

2. An application. We will apply the results of $\S 1$ to discuss existence and uniqueness of continuous solutions to the integral equation

$$
x(t)+\lambda \int_{0}^{t} K(t, s) f(s, x(s)) d s=y(t)
$$


where $K, f, y$ are real valued continuous functions and $\lambda$ a real parameter. We give without proof the nearly obvious

LEMMA 2.1. Let $h(t)$ be any real valued continuous function on $[0, \infty)$. There exists a continuously differentiable function $k(t)$ defined on $[0, \infty)$ satisfying

(i) $k(t) \geqq|h(t)|, t \geqq 0$,

(ii) $k^{\prime}(t) \geqq 1, t \geqq 0$.

We will also need the simple

LEMMA 2.2. Let $x(t)$ be continuous and real valued on $[0, \infty), m(t)$ and $\xi(t)$ real valued, monotone increasing, continuously differentiable and greater than or equal to 1 on $[0, \infty)$. Then, there exists a continuously differentiable function $g(t)$ on $[0, \infty)$ satisfying

(i) $\delta g^{\prime}(t) \geqq \xi(t) m(t) g(t)$,

(ii) $g^{\prime}(t) \geqq|x(t)|$,

(iii) $g(t) \geqq 1$,

for all $t \geqq 0$, where $\delta$ is any given positive number $<1$.

Proof. Let $h(t)=\max \{1,|x(t)|\}$. Apply Lemma 2.1 to obtain $k(t)$ satisfying (i) and (ii) of that lemma. Define

$$
g(t)=\exp \left\{\frac{1}{\delta} \int_{0}^{t} \xi(\tau) m(\tau) k(\tau) d \tau\right\}
$$

To show $g$ has the desired properties is straightforward.

Let $C$ denote the vector space of continuous real valued functions on $[0, \infty)$ with the topology of uniform convergence on compact subsets. Recall $C$ is sequentially complete and locally convex as well as Hausdorff. $C$ is, however, not normable (the fact that $C$ is metrizable we shall not use). We denote the real numbers by $R$.

Define a map $H:(C \times R) \times C \rightarrow C$ by

$$
H(y, \lambda ; x)(t)=x(t)+\lambda \int_{0}^{t} K(t, s) f(s, x(s)) d s-y(t) .
$$

Assume $K$ is continuous on $[0, \infty) \times[0, \infty)$, and let $f$ be continuous on $[0, \infty)$ $\times(-\infty, \infty)$. Assume also that $f$ is Lipschitzian, in the sense that

$$
\left|f\left(t, x_{1}\right)-f\left(t, x_{2}\right)\right| \leqq m(t)\left|x_{1}-x_{2}\right|, \quad t \geqq 0,
$$

for all $x_{1}, x_{2}$, where $m$ is continuously differentiable, monotone increasing and greater than or equal to 1 .

We now construct a bounded covering for $C$. Let $w>0$ and $\delta, 0<\delta<1$, be given. Pick $\delta^{\prime}, 0<\delta^{\prime}<1$, such that $w \delta^{\prime} \leqq \delta$. Define

$$
\begin{aligned}
& \mathscr{E}=\{g \in C: g \text { is continuously differentiable and } \\
& \left.\qquad \delta^{\prime} g^{\prime}(t) \geqq \xi(t) m(t) g(t), t \geqq 0\right\},
\end{aligned}
$$


where $\xi(t)$ is a continuously differentiable monotone increasing function such that

$$
\xi(t) \geqq 1+\sup _{0 \leqq s \leqq t ; 0 \leqq \tau \leqq t}|K(\tau, s)| \geqq 1+\sup _{0 \leqq s \leqq t}|K(t, s)| .
$$

Define

$$
B_{g}=\left\{x \in C:|x(t)| \leqq g^{\prime}(t), t \geqq 0\right\}
$$

for each $g \in \mathscr{E}$. The class $\mathscr{B}$ given by

$$
\mathscr{B}=\left\{\alpha B_{g}: g \in \mathscr{E}, \alpha \geqq 1\right\}
$$

is a bounded covering for $C$, as is easily verified through the use of Lemma 2.2. Find $k, 0<k<1$, satisfying

$$
\int_{0}^{k} \xi(s) m(s) d s<\frac{\delta}{w} .
$$

Fix $\gamma$, an arbitrary positive number, and define

$$
U=\left\{x \in C: \sup _{0 \leqq t \leqq k}|x(t)|<\gamma\right\},
$$

an absolutely convex neighborhood of 0 in $C$. For each $(y, \lambda) \in C \times R$, let $L_{(y, \lambda)}$ be the identity map $I$ on $C$. Finally, let $\varepsilon$ be any positive number and define

$$
P=\left\{x \in C: \sup _{0 \leqq t \leqq n}|x(t)|<\varepsilon\right\},
$$

$n \geqq 1$ an integer, and let $J=(-w, w)$ be an interval in $R$.

With $C \times R=G, C=E=F, H=f, P \times J=K, C=N, U=U_{(y, \lambda)}, \mathscr{B}=\mathscr{B}_{(y, \lambda)}, \delta=\delta_{(y, \lambda)}$ and $I=L_{(y, \lambda)}$ for all $(y, \lambda)$ in $P \times J$, it is only laborious to show that all the hypotheses of Theorem 1.1 and Corollary 1.1 are satisfied, where $y_{0}$ is taken to be $(0,0)$. We shall carry out just a sample computation to indicate the pattern of things.

The condition A3 of Definition 0.2 takes the form: If $B=\alpha B_{g}$ is in $\mathscr{B}$, and $(y, \lambda) \in P \times J$, we need $H\left(y, \lambda ; U \cap \alpha B_{g}\right)-I\left(U \cap \alpha B_{g}\right) \subset H(y, \lambda ; 0)-I(0)+C_{I\left(\alpha B_{g}\right)}$. That is, we need to show that for $x \in U \cap \alpha B_{g}$,

$$
\lambda \int_{0}^{t} K(t, s) f(s, x(s)) d s-y(t) \in \lambda \int_{0}^{t} K(t, s) f(s, 0) d s-y(t)+C_{\alpha B_{g}},
$$

or equivalently, $z(t) \in C_{\alpha B_{g}}$ for all $x \in U \cap \alpha B_{g}$, where

$$
z(t)=\lambda \int_{0}^{t} K(t, s)(f(s, x(s))-f(s, 0)) d s .
$$

But

$$
|z(t)| \leqq|\lambda| \int_{0}^{t}|K(t, s)||m(s)||x(s)| d s \leqq|\lambda| \xi(t) m(t) \int_{0}^{t}|x(s)| d s,
$$

using the monotonicity of $m$ and $\xi$. Since $x \in U \cap \alpha B_{g},|x(s)| \leqq \alpha g^{\prime}(s)$, and so

$$
\begin{aligned}
|z(t)| & \leqq \alpha|\lambda| \xi(t) m(t)(g(t)-g(0)) \\
& \leqq \alpha|\lambda| \xi(t) m(t) g(t) \leqq(\alpha|\lambda| \delta / w) g^{\prime}(t) \leqq \alpha g^{\prime}(t) .
\end{aligned}
$$

Thus, $z(t) \in \alpha B_{g} \subset C_{\alpha B_{g}}$, and A3 is established. 
The Corollary 1.1 yields, then, a unique map $\varphi: M \rightarrow U$ (where $M$ is any neighborhood of $(0,0)$ in $P \times J$ satisfying the condition pointed out in Remark 1.1), such that $H(y, \lambda ; \varphi(y, \lambda))=0$. That is, for each $(y, \lambda) \in M$, there is a unique solution, $\varphi(y, \lambda)$, in $U$ for the equation (2.1). We can, by a judiciously large choice for $\gamma$, ensure that $P \times J$ will do for $M$. But, recall that $P$ and $J$ were themselves quite arbitrary open basic neighborhoods of 0 in their respective spaces. We have nearly completed the proof for

THEOREM 2.1. If $K(t, x)$ and $f(t, x)$ are continuous real valued functions on $[0, \infty) \times[0, \infty)$ and $[0, \infty) \times(-\infty, \infty)$ respectively, and if $f$ satisfies $(2.3)$, then given any $y \in C$ and $\lambda \in R$, the equation (2.1) has a unique, continuous solution $x$ defined on $[0, \infty)$.

Proof. Given any $y \in C, \lambda \in R$, choose $P \times J$ sufficiently large to contain $(y, \lambda)$. The above discussion assures a unique solution, $\varphi(y, \lambda)$, in $U$ when $\gamma$ defining $U$ is taken sufficiently large. If $x_{1}$ were any other solution for the given $(y, \lambda)$, it would be in $U$ also by, perhaps, a larger choice still for $\gamma$, since $U$ is absorbing in $C$. This state of affairs, however, contradicts the uniqueness of the function $\varphi$. Thus, $\varphi(y, \lambda)$ is the only solution corresponding to $(y, \lambda)$ lying in $C$.

REMARK 2.1. It should be remarked that the restriction that the kernel $K$ in (2.1) be continuous (and even that $f$ be continuous) is unnecessary for the argument presented. Any conditions in addition to (2.3) that would insure $\xi(t)$ satisfying (2.5) exists and that $H$ is a continuous map of $(C \times R) \times C$ into $C$ would suffice. The restriction to scalar valued functions can also be relieved. Such modifications are, however, only technical and can easily be investigated by the reader.

The result in Theorem 2.1 is obtainable by other means. A brief discussion with references can be found in [15]. The present result is of interest in that global existence, uniqueness-and, as we shall see below, continuous dependence on parameters-are obtained directly from the iterative construction. Other authors (for example [12] and [13]) have approached the problem by continuing local solutions obtained by iteration, or by employing topological methods for existence and then imposing further restrictions to obtain uniqueness. Corduneanu, [2], has obtained direct global results (not including ours) with an eye to stability theorems by employing methods that bear some similarity to those of our example.

Now, suppose $A$ is any absolutely convex and bounded set in $C$. We may regard $H$ as a mapping of $\left(C_{A} \times R\right) \times C \rightarrow C$, where $C_{A}$ is given its norm topology under $\|\cdot\|_{A}$. Since all the conditions of Theorem 1.2 applying to fixed $(y, \lambda)$ were shown above to hold for $(y, \lambda) \in C \times R$, they a fortiori hold for $(y, \lambda) \in C_{A} \times R$. Still using $I$ as our linear approximation for all $(y, \lambda), W$ is again trivially a neighborhood. Since the norm topology of $C_{A}$ is finer than the topology induced on $C_{A}$ by $C, H$ is easily seen to be continuous on $\left(C_{A} \times R\right) \times C$. Redefining $P$ of $(2.10)$ to be $P=\left\{x \in C_{A}:\|x\|_{A}<\varepsilon\right\}$, the set

$$
S=\left\{I^{-1}(H(y, \lambda ; 0)):(y, \lambda) \in P \times J\right\}
$$


is clearly bounded in $C$. But since $\mathscr{B}$ has the property that every bounded set $B^{\prime}$ in $C$ is contained in some $B \in \mathscr{B}$, it is easy to see that the hypotheses of Theorem 1.2 are all satisfied in this restricted setting. Consequently, the solutions $\varphi(y, \lambda)$ for (2.1), $(y, \lambda) \in C_{A} \times R$, depend continuously on $(y, \lambda)$ for the norm topology of $C_{A}$. We sum up these remarks in

TheOrem 2.2. Let $A$ be an absolutely convex, bounded subset of $C$. For each $(y, \lambda) \in C_{A} \times R$ there exists a unique solution $\varphi(y, \lambda)=x$ of $(2.1)$ in $C$. Further, if $x_{0}=\varphi\left(y_{0}, \lambda_{0}\right)$ is a particular solution, then for each neighborhood $U$ of $x_{0}$ in $C$, there exists $\varepsilon_{A}>0$ such that $\left\|y-y_{0}\right\|_{A}<\varepsilon_{A}$ and $\left|\lambda-\lambda_{0}\right|<\varepsilon_{A}$ implies $\varphi(y, \lambda) \in U$.

3. Differentiability of the implicit function. Throughout this section, we shall adopt some conventions as to notation in order to avoid tedious repetitions. $E$, $F$ and $G$ will denote Hausdorff, locally convex, linear topological spaces. $K \subset G$ and $N \subset E$ will be open subsets containing $0 . f$ will be a map defined on $K \times N$ with values in $F$ satisfying $f(0,0)=0$. For each $y \in K$, we shall denote by $f_{y}$ the map from $N$ to $F$ defined by $f_{y}(x)=f(y, x)$. These partial mappings will be assumed continuous in $N$. $L: E \rightarrow F$ will be a linear homeomorphism which for some $\gamma, 0<\gamma<1$, and some pair-containing bounded covering $\mathscr{B}$ for $E,(\gamma, \mathscr{B})$-approximates every $f_{y}$ at $x=0$ on $U$, an absolutely convex neighborhood of 0 in $E$. The map $g: K \rightarrow F$ defined by $g(y)=f(y, 0)$ will be assumed continuous at $y=0$. It is readily seen that we have assumed enough to apply Corollary 1.1 to deduce the existence of a unique map $\varphi: K^{\prime} \rightarrow U$, where $K^{\prime}$ is an absolutely convex neighborhood of 0 in $K$, and $\varphi$ satisfies $f(y, \varphi(y))=0$. We now proceed to find conditions under which $\varphi$ will be differentiable.

There have been numerous definitions of differentiability proposed for mappings between locally convex spaces over the years (see, for example, [10]). We shall use here essentially the notion of differentiability suggested by Sebastião è Silva [14].

We give now the

Definition 3.1. Let $H$ be an open subset of $G, \mathscr{A}$ a bounded covering for $G$. We will say $\psi: H \rightarrow E$ is differentiable $(\mathscr{A})$ at $y \in H$ if there exists a linear map $T_{y}: G \rightarrow E$, bounded on the sets of $\mathscr{A}$, such that for each $A \in \mathscr{A}$ there is an absolutely convex, bounded set $C_{y}(A) \subset E$ such that for every $\varepsilon>0$, there exists a $\delta_{y}>0$ so that

$$
(\psi(y+t h)-\psi(y)) / t-T_{y} h \in \varepsilon C_{y}(A)
$$

for all $h \in A$ and $t$ satisfying $0<|t|<\delta_{y} . T_{y}$ will be called the derivative $(\mathscr{A})$ of $\psi$ at $y$.

We remark that if $\mathscr{A}$ is a bounded covering for $G$, then the class $\mathscr{A} \times \mathscr{B}$ $=\{A \times B: A \in \mathscr{A}, B \in \mathscr{B}\}$ is a bounded covering for $G \times E$. If $f$ is assumed differentiable $(\mathscr{A} \times \mathscr{B})$, with derivative $T_{y x}$ at $(y, x)$, we will denote by $f_{1 y x}$ and $f_{2 y x}$ the partial mappings defined by

$$
f_{1 y x}(h)=T_{y x}(h, 0) \text { and } f_{2 y x}(k)=T_{y x}(0, k)
$$

for all $h \in G, k \in E$. We are now ready to state our next theorem. 
THEOREM 3.1. Let $\mathscr{A}$ be a bounded covering for $G$ and assume the sets in $\mathscr{B}$ are sequentially closed. Assume that for every $(y, x) \in K \times N, f$ is differentiable $(\mathscr{A} \times \mathscr{B})$ at $(y, x)$ with derivative $T_{y x}$, and that $f_{2 y x}$ is bijective with an inverse that maps bounded sets into bounded sets. Suppose that for each $y \in K$, given $A \in \mathscr{A}$, there is a $B \in \mathscr{B}$ such that

(i) $f(y, 0) \in \frac{1}{2} L(B)$,

(ii) $f_{1 y x}(A)+C_{(y, x)}(A \times B) \subset L(B)$ for all $x \in E_{B} \cap N$, where $C_{(y, x)}(A \times B)$ is as in the definition of differentiability $(\mathscr{A} \times \mathscr{B})$ as applied to $f$ at $(y, x)$.

Then $\varphi$ is differentiable $(\mathscr{A})$ at each $y$ in its domain, and its derivative $(\mathscr{A})$ at $y$ is $-f_{2 y \varphi(y)}^{-1} \circ f_{1 y \varphi(y)}$.

Proof. Fix $y \in K^{\prime}$ (the domain of $\varphi$ ). For any $A \times B \in \mathscr{A} \times \mathscr{B}$, we have, by the differentiability $(\mathscr{A} \times \mathscr{B})$ of $f$ at $(y, 0)$, that for every $\varepsilon>0$, there exists a $\delta_{0}>0$ such that for all $h \in A$,

$$
(f(y+t h, 0)-f(y, 0)) / t-f_{1 y 0}(h) \in \varepsilon C_{(y, 0)}(A \times B)
$$

providing $0<|t|<\delta_{0}$, since, in particular, $0 \in B$. Given a fixed $A \in \mathscr{A}$, find $B$ in accordance with the hypotheses of the theorem. We then conclude, since we may take $\varepsilon<1$, that for all $|t|$ sufficiently small,

$$
(f(y+t h, 0)-f(y, 0)) / t \in f_{1 y 0}(h)+\varepsilon C_{(y, 0)}(A \times B) \subset f_{1 y 0}(A)+C_{(y, 0)}(A \times B) \subset L(B)
$$

for all $h \in A$. Thus,

$$
f(y+t h, 0) \in f(y, 0)+t L(B) \subset L(B),
$$

for all $h \in A$, providing $|t|<\frac{1}{2}$. Consequently, $L^{-1}(f(y+t h, 0))$ is in $B$ for all $h \in A$ if $|t|$ is sufficiently small. Since $B$ is sequentially closed, we can deduce that $\varphi(y+t h) \in B \cap U$ by employing Remark 2 of [11] and recalling that $\varphi$ is the limit of the sequence (1.3). Furthermore, then,

$$
(\varphi(y+t h)-\varphi(y)) / t \in E_{B \cap U}=E_{B} .
$$

In addition, we have

$$
f(y+t h, x)-f(y, x) \in|t| L(B) \text { if } 0<|t|<\delta_{(y, x)}
$$

for each $x \in E_{B} \cap N$. This follows from the differentiability $(\mathscr{A} \times \mathscr{B})$ of $f$ at $(y, x)$ together with (ii) and the fact that $L(B)$ is balanced. For $|t|$ sufficiently small, then,

$$
\begin{aligned}
&\|L(\varphi(y+t h)-\varphi(y))\|_{L(B \cap U)}-\|f(y+t h, \varphi(y+t h))-f(y+t h, \varphi(y))\|_{L(B \cap U)} \\
& \leqq\|f(y+t h, \varphi(y+t h))-f(y+t h, \varphi(y))-L(\varphi(y+t h)-\varphi(y))\|_{L(B \cap U)} \\
& \leqq \gamma\|\varphi(y+t h)-\varphi(y)\|_{B \cap U},
\end{aligned}
$$

using the fact that $L(\gamma, \mathscr{B})$-approximates $f_{y+t h}$ at 0 on $U$. The fact that the second term on the left of the first inequality above is defined follows from (3.2) with $x=\varphi(y)$. We now can conclude, applying Proposition 0.1 , that

$$
\begin{aligned}
(1-\gamma)\|\varphi(y+t h)-\varphi(y)\|_{B \cap U} & \leqq\|f(y+t h, \varphi(y+t h))-f(y+t h, \varphi(y))\|_{L(B \cap U)} \\
& =\|f(y, \varphi(y))-f(y+t h, \varphi(y))\|_{L(B \cap U) .}
\end{aligned}
$$


Since $B$ is bounded, there exists $\alpha \geqq 1$ such that $L(B \cap U) \supset(2 / \alpha) L(B)$. Hence, using (3.2), the last expression above is less than or equal to $(\alpha / 2)|t|, 0<|t|<\delta_{(y, \varphi(y)) \text {. }}$ Thus, for such $t$, we have for all $h \in A$ that

$$
\|(\varphi(y+t h)-\varphi(y)) / t\|_{B \cap U}<\alpha /(1-\gamma)
$$

and consequently that

$$
(\varphi(y+t h)-\varphi(y)) / t \in(\alpha /(1-\gamma)) B .
$$

Let $B^{\prime}=(\alpha /(1-\gamma)) B$. Then $B^{\prime} \in \mathscr{B}$, and for each $h \in A$, there is a $k(h)$ in $B^{\prime}$ such that $\varphi(y+t h)=\varphi(y)+t k(h)$. Since $f$ is differentiable $(\mathscr{A} \times \mathscr{B})$ at $(y, \varphi(y))$, there is an absolutely convex, bounded set $D$ in $F$ such that for every $\hat{\varepsilon}>0$, there exists a $\delta>0$ with

$$
(f(y+t h, \varphi(y)+t k(h))-f(y, \varphi(y))) / t-T_{y \varphi(y)}(h, k(h)) \in \hat{\varepsilon} D
$$

as long as $0<|t|<\delta$, uniformly for all $h \in A, k(h) \in B^{\prime}$. Let $C_{y}(A)=f_{2 y \varphi(y)}^{-1}(D)$. Using the definition of $k(h)$, we can write the last inclusion as

$$
f_{1 y \varphi(y)}(h)+f_{2 y \varphi(y)}((\varphi(y+t h)-\varphi(y)) / t) \in \hat{\varepsilon} f_{2 y \varphi(y)}\left(C_{y}(A)\right) .
$$

From this it easily follows that if $h \in A$ and $|t|$ is taken sufficiently small, then

$$
(\varphi(y+t h)-\varphi(y)) / t-\left(-f_{2 y \varphi(y)}^{-1} \circ f_{1 y \varphi(y)}\right)(h) \in \hat{\varepsilon} C_{y}(A) .
$$

Hence, $\varphi$ is differentiable $(\mathscr{A})$ at $y$.

\section{REFERENCES}

1. R. G. Bartle, Implicit function theory in groups, Math. Z. 62 (1955), 335-346.

2. C. Corduneanu, Problèmes globaux dans la théorie des équations intégrales de Volterra, Ann. Mat. Pura Appl. (4) 67 (1965), 349-363. MR 32 \#331.

3. J. Dieudonné, Foundations of modern analysis, Pure and Appl. Math., vol. 10, Academic Press, New York, 1960. MR 22 \#11074.

4. E. Dubinsky, Fixed points in non-normed spaces, Ann. Acad. Sci. Fenn. Ser. A. I. No. 331 (1963). MR $27 \# 1801$.

5. P. L. Falb and M. Q. Jacobs, On differentials in locally convex spaces, J. Differential Equations 4 (1968), 444-459. MR 37 \#5696.

6. H. R. Fischer, Differentialkalkül für nicht-metrische Strukturen, Ann. Acad. Sci. Fenn. A. I. No. 247 (1957). MR 19, 869.

7. A. Frölicher and W. Bucher, Calculus in vector spaces without norm, Lecture Notes in Math., no. 30, Springer-Verlag, Berlin and New York, 1966. MR 35 \#4723.

8. J. Gil de Lamadrid, Topology of mappings and differentiation processes, Illinois J. Math. 3 (1959), 408-420. MR 21 \#5918.

9. J. Horváth, Topological vector spaces and distributions. Vol. I, Addison-Wesley, Reading, Mass., 1966. MR 34 \#4863.

10. H. H. Keller, Differenzierbarkeit in topologischen Vektorraümen, Comment. Math. Helv. 38 (1964), 308-320. MR 29 \#3858.

11. T. S. McDermott, Nonlinear mappings in locally convex spaces, Trans. Amer. Math. Soc. 153 (1971), 157-165. 
12. R. K. Miller and G. R. Sell, Existence, uniqueness and continuity of solutions of integral equations, Ann. Mat. Pura Appl. (4) 80 (1968), 135-152. MR 40 \#663.

13. T. Satō, Sur l'équation intégrale non linéaire de Volterra, Compositio Math. 11 (1953), 271-290. MR 15, 714.

14. J. Sebastião è Silva, Le calcul différentiel et intégral dans les espaces localement convexes. I, Atti Accad. Naz. Lincei Rend. Cl. Sci. Fis. Mat. Nat. (8) 20 (1956), 753-760. MR 19, 561.

15. A. Strauss, On a perturbed Volterra integral equation, J. Math. Anal. Appl. 30 (1970), 564-575.

University of Hawail, Honolulu, Hawail 96822 